\title{
LncRNA expression profiles and validation in keloid and normal skin tissue
}

\author{
XUEBING LIANG $^{1 *}$, LIN MA $^{2 *}$, XIAO LONG ${ }^{1}$ and XIAOJUN WANG ${ }^{1}$ \\ Divisions of ${ }^{1}$ Plastic Surgery and ${ }^{2}$ Surgery, Peking Union Medical College Hospital, Peking Union Medical College, \\ Chinese Academy of Medical Sciences, Beijing 100730, P.R. China
}

Received July 14, 2015; Accepted September 4, 2015

DOI: $10.3892 /$ ijo.2015.3177

\begin{abstract}
Keloid is a type of pathological skin scar. Pathogenesis of keloid is complex and is not fully understood. LncRNA can regulate gene expression on different levels. It also participates in cell cycle regulation and cell proliferation. The present study investigated the potential biological function of lncRNA in keloid. We identified differential expression of lncRNAs and mRNAs between 3 pairs of keloid and normal skin tissue by microarray. Differentially expressed lncRNAs were validated by quantitative reverse transcriptase-PCR (qRT-PCR). Gene Ontology (GO) and pathway analysis presented the characteristics of associated protein-coding genes. Additionally, a co-expression network of IncRNA and mRNA was constructed to find potential underlying regulation targets. There were 1,731 lncRNAs constantly upregulated and 782 downregulated, 1,079 mRNAs upregulated and 3,282 downregulated in keloid respectively (fold change $\geq 2.0, \mathrm{P}<0.05$ ). We chose, respectively, 3 upregulated and 1 downregulated lncRNA for qRT-PCR and results were consistent with microarray. Moreover, 11 pathways were related with upregulated transcripts and 44 with downregulated in keloid. The co-expression network revealed that one IncRNA was connected with numerous mRNAs, and vice versa. Furthermore, bioinformation analysis suggested that lncRNA CACNA1G-AS1 may be crucial to keloid formation. In conclusion, groups of lncRNAs were aberrantly expressed in keloid compared with normal skin tissue, which indicated that differentially expressed lncRNAs may play a key role in keloid formation. The present study provides new insights into keloid pathology and potential targets for treatment of keloid.
\end{abstract}

Correspondence to: Dr Xiaojun Wang, Division of Plastic Surgery, Peking Union Medical College Hospital, Peking Union Medical College, Chinese Academy of Medical Sciences, No. 1 Dongdan Shuaifuyuan, Dongcheng, Beijing 100730, P.R. China

E-mail: wangxjplastic@sina.com

*Contributed equally

Key words: keloid, lncRNA, gene expression, calcium channel, CACNA1G-AS1

\section{Introduction}

Keloid is a benign tumor with characteristics of hyperproliferation of fibroblasts and excessive accumulation of extracellular matrix. This pathological process is often triggered by a skin injury. The lesion invades into the normal skin , and forms a solid nodule which is unable to regress spontaneously (1). Multiple therapeutic methods have been reported, such as surgery, radiotherapy, hormone injection, intense pulsed light (IPL) and intralesional cryotherapy $(2,3)$. However, long-term follow-up has shown a relative high rate of recurrence (4). New therapies have also been applied recently, while safety and compared effect need to be further examined $(5,6)$. To better understand the disease, multiple studies have been conducted to explore the complex pathological mechanism. Nakashima et al (7) identified 4 single nucleotide polymorphism (SNP) loci related to keloid by a multistage genome-wide association study in the Japanese population. Chen et al (8) found 250 upregulated and 152 downregulated genes in keloid compared with normal skin. Moreover, several signaling pathways and transcriptional factors are found associated $(9,10)$. However, the precise linkage gene and pathological process are still controversial.

Long non-coding RNA (lncRNA) refers to a class of noncoding RNA, with length more than 200 nt. LncRNA has been confirmed vital to genomic imprinting, dosage compensation, pluripotency-regulation and organism development $(11,12)$. Although there are no reports on IncRNA in keloid, it does play an important role in skin homeostasis and diseases $(13,14)$. For example, IncRNA such as anti-differentiation non-coding RNA (ANCR) and terminal differentiation-induced non-coding RNA (TINCR) are essential for epidermal stability (15). A deletion of ANCR induces epidermal differentiation spontaneously (16).

Considering that lncRNA has a major role in both normal biological growth and pathological development of diseases, we hypothesize it may also participate in keloid formation. In the present study, we adopted high-throughput microarray screening to compare the differences of lncRNA and mRNA expression profiles between keloid and normal skin tissues. Quantitative RT-PCR followed to confirm the results from microarray. By bioinformation analysis, we used the candidate lncRNAs and their potential related protein-coding genes to construct a coding-non-coding gene co-expression diagram. 


\section{Materials and methods}

Patients and samples. The present study was approved by the Ethics Committee Board of the Chinese Academy of Medical Sciences and Peking Union Medical College. All the patients involved in this program gave informed consent to the work. We obtained keloid and corresponding normal skin from 16 patients who received surgery in Peking Union Medical College Hospital. None of the patients had received previous treatment for keloid. The diagnosis of keloid was confirmed by the pathological results. During the surgery, keloid was excised completely, and normal skin tissue was obtained after trimming. Tissue was frozen by liquid nitrogen and stored at $-80^{\circ} \mathrm{C}$.

Total RNA extraction. Total RNA was extracted from the frozen tissue using a TRIzol reagent (Invitrogen, Carlsbad, CA, USA). Each step followed the manufacturer's instructions (Invitrogen). The RNA quality was assessed by NanoDrop ND-1000 (Thermo Fisher Scientific, Waltham, MA, USA), and RNA integrity was assessed by standard denaturing agarose gel electrophoresis.

Microarray profiling. Keloid and normal skin tissue from 3 randomly selected patients were tested in microarray profiling. We used the ArrayStar Human LncRNA/mRNA Expression Microarray version 3.0 (Arraystar, Inc., Rockville, MD, USA). In total, 30,586 lncRNAs and 26,109 coding transcripts can be detected by this microarray. Public transcriptome databases and IncRNA publications were used to construct IncRNAs.

RNA labeling and array hybridization. Sample labeling and array hybridization were performed according to the Agilent One-Color Microarray-Based Gene Expression Analysis protocol (Agilent Technologies) with minor modifications. First, mRNA was purified from total RNA after removal of rRNA (mRNA-ONLY ${ }^{\mathrm{TM}}$ Eukaryotic mRNA isolation kit; Epicentre, Madison, WI, USA). Then, each sample was amplified and transcribed into fluorescent cRNA along the entire length of the transcripts without 3' bias utilizing a random priming method (Quick Amp Labeling kit, One-Color; Agilent $\mathrm{p} / \mathrm{n}$ 5190-0442). The labeled cRNAs were purified by RNeasy Mini kit (Qiagen p/n 74104). The concentration and specific activity of the labeled cRNAs (pmol Cy3/ $/ \mu \mathrm{g}$ cRNA) were measured by NanoDrop ND-1000. Hybridization adopted Agilent Gene Expression Hybridization kit (Agilent p/n 51885242). A total of $1 \mu \mathrm{g}$ of each labeled cRNA was fragmented by adding $5 \mu 110 \mathrm{X}$ blocking agent (Agilent $\mathrm{p} / \mathrm{n}$ 5188-5242) and $1 \mu \mathrm{l}$ of $25 \mathrm{X}$ fragmentation buffer (Agilent $\mathrm{p} / \mathrm{n}$ 5188-5242), then the mixture was heated at $60^{\circ} \mathrm{C}$ for $30 \mathrm{~min}$, finally $25 \mu \mathrm{l} 2 \mathrm{X}$ GE hybridization buffer (Agilent p/n 5188-5242) was added to dilute the labeled cRNA. A total of $50 \mu \mathrm{l}$ of hybridization solution was dispensed into the gasket slide and assembled to the lncRNA expression microarray slide. The slides were incubated for $17 \mathrm{~h}$ at $65^{\circ} \mathrm{C}$ in an Agilent hybridization oven (Agilent p/n G2545A). The hybridized arrays were washed, fixed and scanned using the Agilent DNA G2505C microarray.

Data analysis. Agilent Feature Extraction software (version 11.0.1.1) was used to examine acquired array images. Quantile normalization and subsequent data processing were executed using the GeneSpring GX v11.5.1 software package (Agilent Technologies). After quantile normalization of the raw data, lncRNAs and mRNAs, at least 3 out of 6 samples, were chosen for further data analysis. Differentially expressed lncRNAs/mRNAs were classified by a fold change cut-off of 2.0 (upregulated or downregulated) combined with P-value $<0.05$ as selection criterion. The microarray analysis was performed by Kangcheng Biology Engineering Co., Ltd., (Shanghai, China).

GO and pathway analysis. The Gene Ontology (GO) project offers a controlled vocabulary to label gene and gene product attributes in any organism (http://www.geneontology.org). Three fields are covered in the ontology, which are biological process (BP), cellular component (CC) and molecular function (MF). To avoid more than accidental overlap between the DE list and the GO annotation list exists, Fisher's exact test is applied. P-value represents the significance of GO terms enrichment in the DE genes. Pathway analysis is a functional analysis mapping genes to KEGG pathways. The P-value (EASE-score, Fisher-P value or Hypergeometric-P-value) indicates the significance of the pathway correlated to the conditions. The lower the P-value, the more significant is the pathway. The analysis was performed by Kangcheng Biology Engineering.

The coding-non-coding gene co-expression network. We chose 4 lncRNAs (uc002lfu.1, ENST00000522743, NR_038439 and ENST00000521141) to explore the connection between these lncRNAs and corresponding coding genes. First, the median value of all the transcripts expressed from the same coding gene was selected, while there was no special treatment with the expression value of lncRNA. Secondly, differentially expressed lncRNAs and mRNAs data were screened and taken away from dataset. Thirdly, Pearson correlation coefficient (PCC) between coding gene and IncRNA was calculated using R-value. Fourthly, PCC $\geq 0.999$ was chosen as meaningful related pair. Finally, the network was drawn through Cytoscape (v2.8.1). In the network, we used blue nodes representing coding genes, and red/pink nodes representing lncRNAs. A solid line indicates a positive correlation, and a dashed line a negative correlation.

Quantitative real-time PCR and statistical methods. We used quantitative real-time PCR (qRT-PCR) to confirm the expression levels. Total RNA was extracted from frozen tissue using a TRIzol reagent (Invitrogen Life Technologies) and reversetranscribed using RT reagent kit (Thermo Fisher Scientific) according to the manufacturer's instructions. Real-time PCR was performed using the SYBR-Green method with ViiA 7 real-time PCR system (Applied Biosystems) following the manufacturer's protocols. Three significantly upregulated lncRNAs (uc002lfu.1, ENST00000522743 and NR_038439) and one downregulated lncRNAs (ENST00000521141) were chosen to test the reproducibility of the data. After further research, NR_038439 drew our attention and was tested in 13 other pairs of tissues. Glyceraldehyde three-phosphate dehydrogenase (GAPDH) acted as endogenous control. Primer sequences were as follows: GAPDH: forward, 5'-GGGAAA 

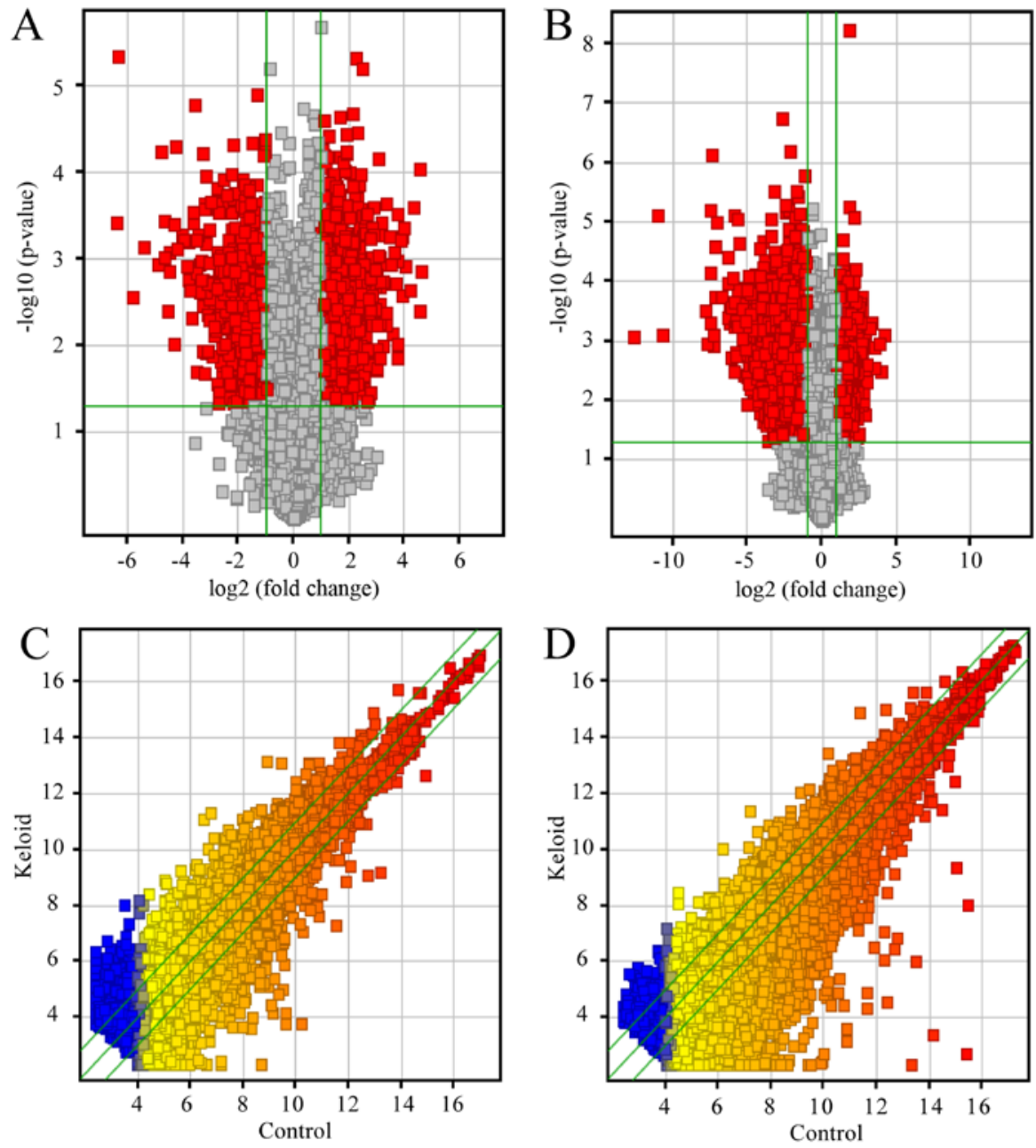

Figure 1. Profiles of lncRNA and mRNA microarray data. Volcano plots of lncRNA expression profiles (A) and mRNA expression profiles (B) are for visualizing differential expression between two different conditions. The red point in the plot represents differentially expressed RNAs with statistical significance. The vertical lines stand for 2.0-fold up and down and the horizontal line indicates P-value of 0.05. The scatter-plots of lncRNA (C) and mRNA (D) are used for assessing expression variation between keloid and normal skin tissue. The values of the $\mathrm{x}$ and $\mathrm{y}$-axes in the scatter plot are the averaged normalized signal values of the group ( $\log 2$ scaled). The default fold change given is 2.0 .

CTGTGGCGTGAT-3' and reverse, 5'-GAGTGGGTGTCG CTGTTGA-3'; uc002lfu.1: forward, 5'-TGCTTGATCCAAA TAATGCC-3' and reverse, 5'-TTCAGTCCAGAGATGTG CC-3'; ENST00000522743: forward, 5'-AGACCCAAAGCT GACACCA-3' and reverse, 5'-ATCTCCCCTCATCCAAA CC-3'; NR_038439: forward, 5'-CTGGCAAGGTTGAGTA GGCT-3' and reverse, 5'-TGCTCCCTTCACACGGTCA-3'; ENST00000521141: forward, 5'-AAGGATGTGGGAGTTT GAGAC-3' and reverse, 5'-CAGAGGGGAGAGCGTGTTC-3'. The PCR conditions were as follows: PCR reaction under $95^{\circ} \mathrm{C}$ for $10 \mathrm{~min}$, then 40 cycles of $95^{\circ} \mathrm{C}$ for $10 \mathrm{sec}$ and $60^{\circ} \mathrm{C}$ for $60 \mathrm{sec}$. After setting the concentration of PCR product as 1, all the PCR products were diluted as follows: $1 \times 10^{-1}, 1 \times 10^{-2}$, $1 \times 10^{-3}, 1 \times 10^{-4}, 1 \times 10^{-5}, 1 \times 10^{-6}, 1 \times 10^{-7}, 1 \times 10^{-8}$ and $1 \times 10^{-9}$. As the target genes and housekeeping gene went through PCR separately, the standard curves were drawn based on the dilution. The relative expression (RQ) was obtained through dividing the concentration of target gene by the one of housekeeping gene. The lncRNA expression differences between keloid and normal skin were analyzed using Student's t-test with SPSS (version 16.0; SPSS, Inc., Chicago, IL, USA). A value of $\mathrm{P}<0.05$ was considered significant.

\section{Results}

Differentially expressed lncRNAs between keloid and normal skin tissues. The microarray we used in the present study can detect 30,586 lncRNAs and 2,6108 annotated mRNAs from authoritative data sources including 'RefSeq', 'UCSC knowngene' and 'Genecode'. We found a total of 16,710 lncRNAs expressed in keloid from lncRNA expression profiling data using microarray analysis (Fig. 1A). Through these data, we compared lncRNA expression levels among 3 pairs of human keloid and their adjacent normal skin tissue (Fig. 1C). There were an average of 2,988 upregulated IncRNAs (range from 2,885 to 3,071 ) and 1,152 downregulated IncRNAs (range from 1,036 to 1,316 ), which were significantly differentially expressed ( $\geq 2.0$-fold) (Fig. 2A). To make the results more precise and reduce the possibility of false positive, we set the screen standard of lncRNA as follows: The abnormal lncRNA should exist in all 3 pairs of tissues. After screening, there were 1,731 constantly upregulated lncRNAs and 782 downregulated lncRNAs in keloid compared with normal skin. Top 25 differentially expressed lncRNAs are listed in Table I. In the upregulated IncRNAs, the maximum fold change was 

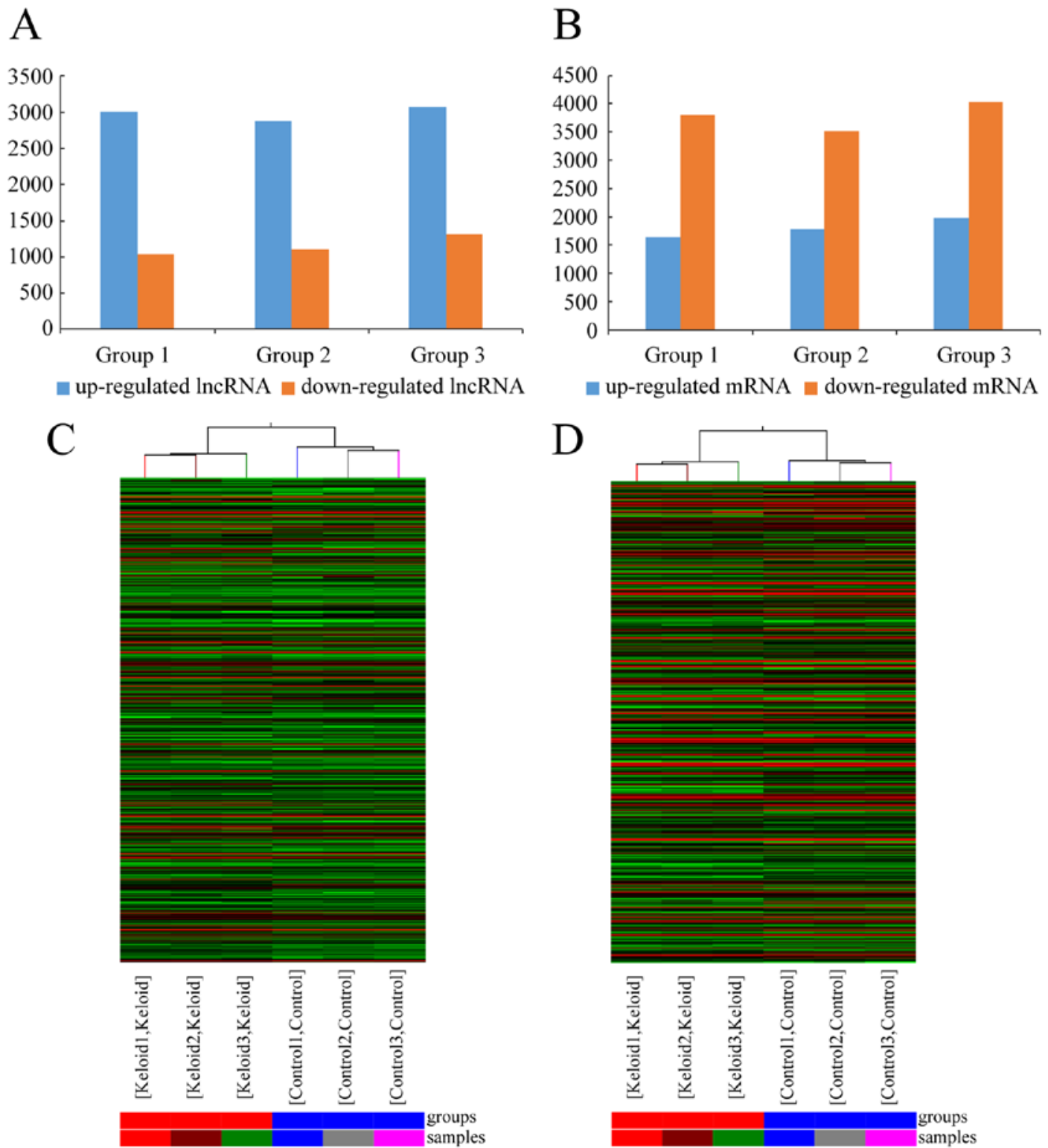

Figure 2. The differentially expressed profiles between keloid and normal skin. The number of upregulated and downregulated lncRNAs (A) varied across the three pairs of samples, which is the same in mRNAs (B). Hierarchical clustering of lncRNA (C) and mRNA (D) were used for analysis of gene expression data. Cluster analysis arranges samples into groups according to their expression level, which allows to form hypothesis of the relationships among samples. Each column represents a sample and each row represents a gene. 'Red' indicates high relative expression, and 'green' indicates low relative expression.

24.68, which belonged to uc002lfu.1. Moreover, among the downregulated lncRNAs, ENST00000449151 possessed the maximum fold change, which was 84.18 . The dendrogram from hierarchical clustering analysis exhibits relationships among lncRNA expression patterns (Fig. 2C).

Differentially expressed mRNAs between keloid and normal skin tissues. The mRNA expression profiling data showed a total of 18,788 mRNAs in keloid using microarray analysis (Fig. 1B). After comparing the three keloids with their paired adjacent normal skin tissue (Fig. 1D), we found an average of 1,799 upregulated mRNAs (range from 1,643 to 1,975) and 3,784 downregulated mRNAs (range 3,516 from 4,035) which were significantly differentially expressed (fold change $\geq 2.0$ ) (Fig. 2B). In the three paired samples, 1,079 mRNAs were consistently upregulated and 3,282 were consistently downregulated. Top 25 differentially expressed mRNAs are listed in Table II. In the upregulated mRNAs, NM_003014 possessed the maximum fold change, which was 18.22. In the downregulated mRNAs, NM_053283 had the maximum fold change, which was 6366.20. The clustering analysis showed relationships among the mRNA expression patterns, which were present in the samples (Fig. 2D).

GO analysis and pathway analysis. GO analysis was used to analyze the main function of the closest coding genes according to the GO database. We found that in upregulated transcripts fromkeloid, the highest enriched GOs were biological regulation (biological process) (Fig. 3A), membrane (cellular component) (Fig. 3B), and receptor activity (molecular function) (Fig. 3C). Also, in the downregulated transcripts, the highest enriched GOs were cellular process (biological process) (Fig. 3D), 
Table I. Top 25 differentially expressed lncRNAs with >2-fold change in 3 paired keloid (K) compared with normal skin tissue (N).

\begin{tabular}{|c|c|c|c|c|c|}
\hline \multicolumn{3}{|c|}{ Upregulated lncRNAs } & \multicolumn{3}{|c|}{ Downregulated lncRNAs } \\
\hline Seqname & $\mathrm{P}$-value & $\begin{array}{c}\text { Absolute fold } \\
\text { change (K vs. } \mathrm{N})\end{array}$ & Seqname & $\mathrm{P}$-value & $\begin{array}{l}\text { Absolute fold change } \\
\qquad(\mathrm{K} \text { vs. } \mathrm{N})\end{array}$ \\
\hline uc002lfu.1 & 0.001392141 & 24.67823539 & ENST00000449151 & 0.000373091 & 84.17830568 \\
\hline ENST00000448264 & $9.24902 \mathrm{E}-05$ & 23.71568882 & ENST00000504509 & $4.5935 \mathrm{E}-06$ & 80.75090928 \\
\hline ENST00000522743 & 0.003964706 & 23.32120992 & TCONS_00014447 & 0.002743683 & 55.75905607 \\
\hline NR_002785 & 0.000245806 & 19.67171045 & ENST00000508884 & 0.000718046 & 43.04190122 \\
\hline ENST00000563754 & 0.002278823 & 18.09086601 & ENST00000543512 & 0.001157162 & 29.62729347 \\
\hline ENST00000568031 & 0.001182026 & 16.43215739 & NR_037166 & $5.8668 \mathrm{E}-05$ & 27.86637043 \\
\hline ENST00000430468 & 0.000440694 & 15.99398905 & NR_049776 & 0.000356012 & 25.44496144 \\
\hline ENST00000568767 & 0.002652791 & 15.40462545 & ENST00000507950 & 0.00097244 & 25.35162231 \\
\hline ENST00000564832 & 0.000561264 & 15.3230045 & ENST00000432377 & 0.004085284 & 23.58314193 \\
\hline ENST00000524045 & 0.000617583 & 14.35734021 & NR_046258 & 0.000818479 & 22.51173429 \\
\hline NR_046438 & 0.001061987 & 13.5487176 & NR_046259 & 0.001389504 & 22.22674064 \\
\hline ENST00000514468 & 0.009747714 & 13.40050409 & ENST00000425399 & 0.009559389 & 19.76933951 \\
\hline ENST00000439186 & 0.000298858 & 13.26738526 & uc002nrq.3 & 0.000394509 & 19.66993157 \\
\hline ENST00000399342 & 0.014008237 & 13.24836573 & ENST00000524012 & $4.96526 \mathrm{E}-05$ & 19.12630922 \\
\hline ENST00000440570 & 0.001883674 & 12.84056697 & uc001gzl.3 & 0.000755965 & 18.64074653 \\
\hline ENST00000444114 & 0.000827626 & 12.38834219 & ENST00000565308 & 0.000520998 & 16.33469184 \\
\hline ENST00000518932 & 0.007291264 & 11.83886059 & NR_034059 & 0.000590883 & 15.93734199 \\
\hline NR_038439 & 0.001173376 & 11.63954238 & ENST00000529667 & 0.001227514 & 14.76394367 \\
\hline ENST00000425711 & 0.000554642 & 11.11258257 & TCONS_00020843 & 0.001779851 & 13.8754485 \\
\hline NR_033997 & 0.001949956 & 10.53634973 & TCONS_00005194 & 0.000640191 & 13.76677473 \\
\hline ENST00000438154 & 0.005762697 & 10.15674579 & ENST00000513915 & 0.002284575 & 13.24861031 \\
\hline AL512723 & 0.004287107 & 10.09486468 & uc001uum.3 & 0.00484692 & 12.76120477 \\
\hline ENST00000424094 & 0.000224946 & 9.997377279 & ENST00000521141 & 0.000288334 & 12.44615316 \\
\hline ENST00000489520 & 0.004207864 & 9.470087791 & ENST00000521188 & 0.00038272 & 12.39901463 \\
\hline TCONS_00023486 & 0.001831401 & 9.387344686 & NR_027856 & 1.6202E-05 & 12.11977043 \\
\hline
\end{tabular}

P-values $<0.05$ are considered significant.

cell part/cell (cellular component) (Fig. 3E), and binding (molecular function) (Fig. 3F). In pathway analysis, there were a total of 11 pathways related with upregulated transcripts in keloid (Fig. 4A). The most enriched network was Neuroactive ligand-receptor interaction, Homo sapiens (human). Also, 44 pathways were found related with downregulated transcripts in keloid (Fig. 4B). Among them, the most enriched network was valine, leucine and isoleucine degradation, Homo sapiens (human). It is interesting that calcium pathway signaling was one of the items above. Considering calcium played a vital role in wound healing, a common process in keloid formation and calcium ion influx inhibitor was used for keloid treatment (17), calcium pathway signaling was important in pathological behavior.

Construction of the co-expression network. We built a co-expression network using correlation analysis between differentially expressed lncRNAs (uc002lfu.1, ENST00000522743, NR_038439, ENST00000521141) and mRNAs (Fig. 5). LncRNAs and mRNAs with Pearson correlation coefficients not less than 0.999 were chosen to draw the network by Cytoscape (v2.8.1). There were 4 lncRNAs and 298 mRNAs in this network, 302 nodes possessed 304 pairs of connections between lncRNAs and mRNAs. The network was complex and indicated that one lncRNA was associated with multiple mRNAs, and vice versa.

Validation of the microarray finding by $q R T-P C R$. In order to validate the results from microarray, we selected 3 upregulated (uc002lfu.1, ENST00000522743 and NR_038439) and 1 downregulated IncRNA (ENST00000521141) from the differentially expressed lncRNA profile, then performed quantitative RT-PCR in original tissues. All of the chosen lncRNAs exhibited abundant expression and had significant changes between the two groups in microarray. The results of qRT-PCR were consistent with microarray data (Fig. 6A). Because calcium signaling pathway may be related with keloid, we chose NR_038439 (IncRNA CACNA1G-AS1), an antisense RNA to CACNA1G which encodes a subtype of T-type $\mathrm{Ca}^{2+}$ channels, and performed qRT-PCR in another 13 pairs of examples. The result was consistent $(\mathrm{P}<0.05)$ (Fig. 6B). 


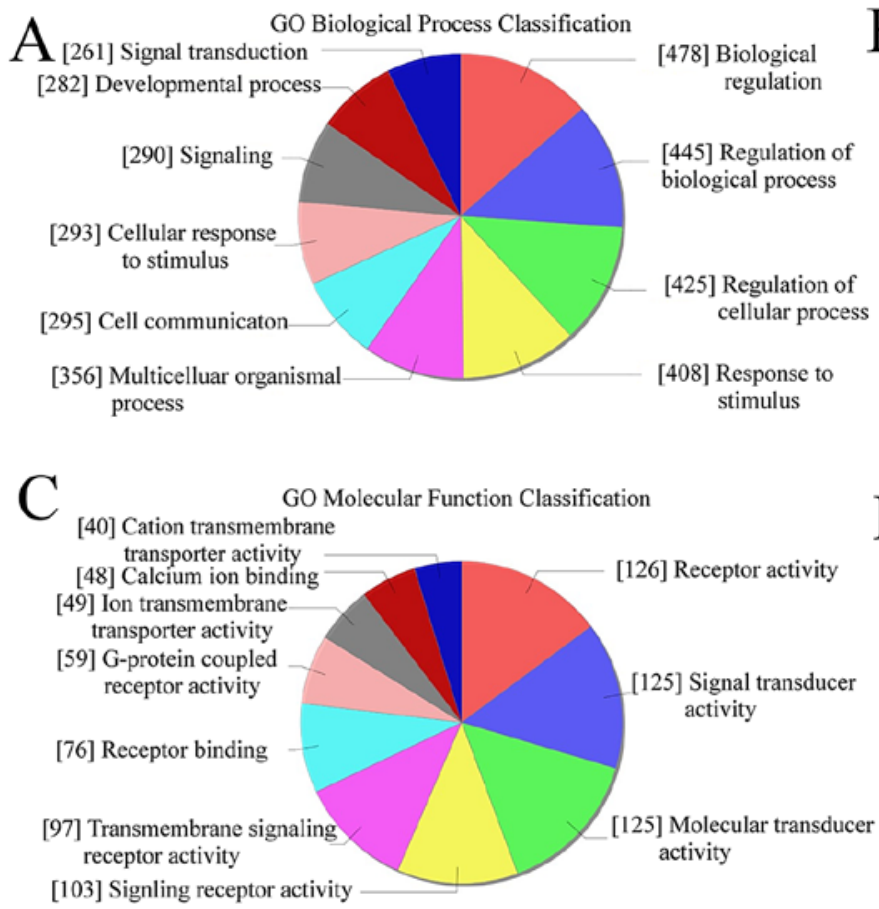

$\mathrm{B}$

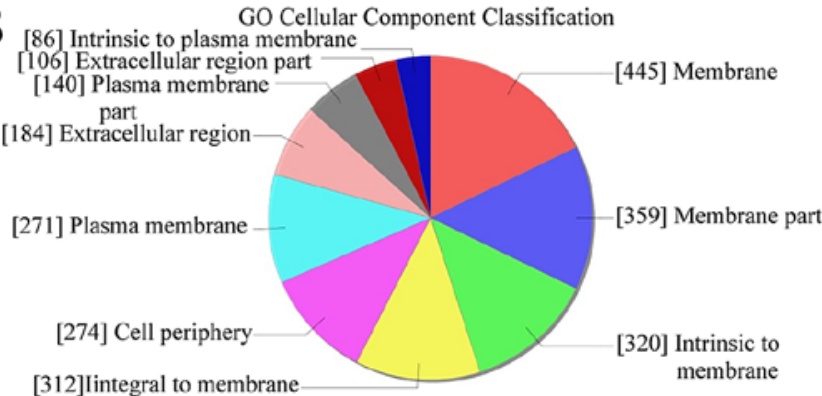

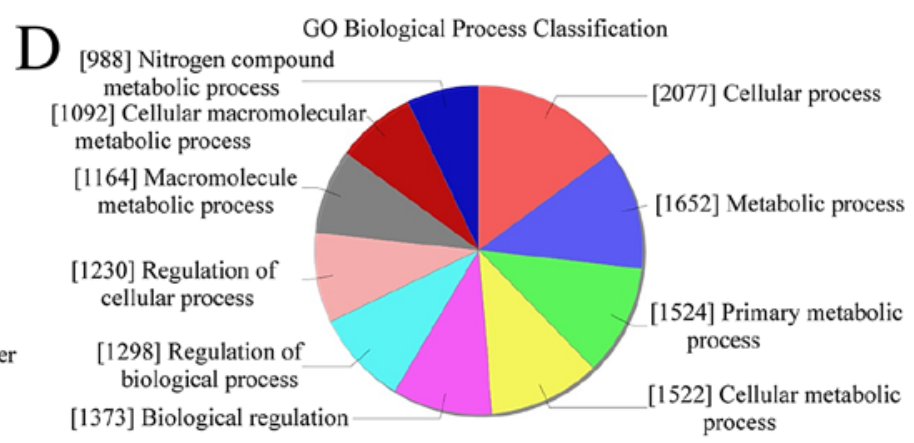
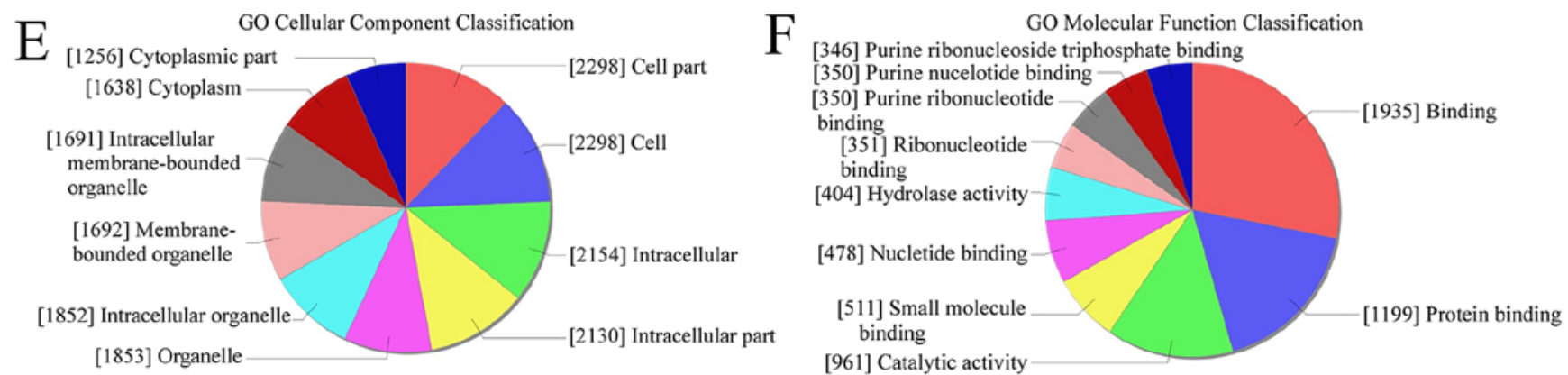

Figure 3. The Gene Ontology analysis. The results show differently expressed coding gene transcripts associated with the biological process (BP), cellular components (CC) and molecular function (MF). Highest enriched GOs targeted by upregulated transcripts in keloid include (A) biological process (BP), (B) cellular component (CC) and (C) molecular function (MF). Highest enriched GOs targeted by downregulated transcripts in keloid include (D) biological process (BP), (E) cellular component (CC) and (F) molecular function (MF). Top 10 items are shown.

\section{Discussion}

In the present study, we have identified a group of lncRNAs and mRNAs, which had abnormal expression in keloid. To the best of our knowledge, this is the first large-scale lncRNA screening, and study of its role in keloid. Non-coding RNA has been investigated in keloid, and there are many exciting reports (18). Liu et al (19) used miRNA microarray in 13 couples of keloid and normal skin, and a total of 32 miRNAs were found with abnormal expression in keloid. They also found that miR-21 could affect apoptosis of keloid fibroblast by negatively regulating the expression of PTEN (20). Compared with microRNA, lncRNA is larger, and has more complex structure. Deregulated expression of lncRNA will disrupt cellular physiology, and then lead to pathologies (21). Thus, we presumed that it could be vital to the formation of keloid.

LncRNAs are important in both skin homeostasis and diseases. When TINCR is deficient, epidermis is found with abnormal morphological characteristics by absence of keratohyalin granules and lamellar bodies, which are essential for protecting skin from environmental damage (22). On the other hand, IncRNAs are also crucial in cutaneous malignancies. Vitamin D and its receptor (VDR) are indispensable for preventing epidermal tumor (23), partially due to VDR keeping a balance between oncogenic lncRNAs and tumor suppressors (24). After comparing lncRNAs in melanoma cell lines, melanocytes and keratinocytes, SPRY4-IT1 was found upregulated in melanoma cells (25). Following knockdown, the invasion and proliferation of melanoma ceased, and the apoptosis of the cells increase. Mazar and his colleagues found that Lipin 2 was a binding protein to SPRY4-IT1 (26). They proposed that an abnormal expression of SPRY4-IT1 led to an increased level of Lipin 2, which may cause the disease. The mechanism of how lncRNAs function has not been fully studied, yet several models have been proposed. LncRNAs can perform as molecular signals, decoys, guiding certain protein to specific location and scaffolds (27). Therefore, further 
Table II. Top 25 differentially expressed mRNAs with >2-fold change in 3 paired keloid (K) compared with normal skin tissue (N).

\begin{tabular}{|c|c|c|c|c|c|}
\hline \multicolumn{3}{|c|}{ Upregulated lncRNAs } & \multicolumn{3}{|c|}{ Downregulated lncRNAs } \\
\hline Seqname & P-value & $\begin{array}{c}\text { Absolute fold } \\
\text { change (K vs. } \mathrm{N})\end{array}$ & Seqname & P-value & $\begin{array}{c}\text { Absolute fold } \\
\text { change (K vs. } N)\end{array}$ \\
\hline NM_003014 & 0.000766034 & 18.22751605 & NM_053283 & 0.000868472 & 6366.203092 \\
\hline ENST00000437936 & 0.00312955 & 16.04025716 & NM_002652 & $7.5475 \mathrm{E}-06$ & 2026.938265 \\
\hline NM_207373 & 0.001108182 & 14.5670146 & NM_002411 & 0.000770676 & 1643.833883 \\
\hline NM_001010876 & 0.00288386 & 11.99403154 & NM_003251 & 0.000312511 & 220.2447826 \\
\hline ENST00000368789 & 0.001235475 & 11.63268318 & NM_152310 & 0.001096438 & 208.8875889 \\
\hline NM_001168243 & 0.000492872 & 9.6787663 & NM_001080526 & 0.000480409 & 176.4587386 \\
\hline NM_005824 & 0.000601708 & 9.311818897 & ENST00000235547 & 6.3784E-06 & 174.2506749 \\
\hline ENST00000354373 & 0.000601688 & 9.000401956 & NM_015973 & 6.96809E-05 & 173.3800147 \\
\hline ENST00000370532 & 0.004754361 & 8.164392456 & ENST00000312150 & $7.50899 \mathrm{E}-07$ & 172.9050825 \\
\hline ENST00000359320 & 0.002391107 & 7.870021989 & NM_031962 & 0.000808876 & 154.1275941 \\
\hline NM_001135 & 0.000714446 & 7.627240018 & NM_181535 & 0.001212281 & 150.8484539 \\
\hline NM_001145143 & 0.002748101 & 7.609122032 & NM_007008 & 0.000180465 & 148.9725592 \\
\hline NM_001199 & 0.003800958 & 7.513524934 & ENST00000301656 & $2.65207 \mathrm{E}-05$ & 146.1537903 \\
\hline NM_012364 & 0.017102348 & 7.281585238 & NM_033185 & $9.90898 \mathrm{E}-06$ & 131.6408041 \\
\hline NM_001159709 & 0.010406205 & 6.717817641 & NM_001482 & 0.000181169 & 106.3194866 \\
\hline NM_032643 & 0.001126809 & 6.638854499 & NM_033187 & 0.000107453 & 77.30322869 \\
\hline ENST00000435607 & 0.007040186 & 6.635851494 & NM_198692 & $3.8766 \mathrm{E}-05$ & 76.91933758 \\
\hline NM_176891 & 0.001531053 & 6.567488034 & NM_031964 & 0.000399172 & 76.61683722 \\
\hline ENST00000389623 & 0.000309148 & 6.508019919 & NM_199161 & 0.000242353 & 72.38119393 \\
\hline NM_018250 & 0.001095794 & 6.407558892 & NM_145792 & 0.000497027 & 71.81391287 \\
\hline NM_001005466 & 0.002540038 & 6.395862838 & ENST00000360770 & 0.00026272 & 70.84177719 \\
\hline NM_005651 & 0.01139665 & 6.349965704 & NM_030754 & 0.001857474 & 67.74012607 \\
\hline ENST00000319331 & 0.000179028 & 6.191758346 & ENST00000182377 & 8.80738E-05 & 62.45728419 \\
\hline NM_203371 & 0.000499894 & 6.113256778 & NM_001123387 & 0.003119069 & 61.24489543 \\
\hline NM_001167916 & 0.004378669 & 6.091668213 & ENST00000368744 & 0.000219518 & 59.85728897 \\
\hline
\end{tabular}

P-values $<0.05$ is considered significant.

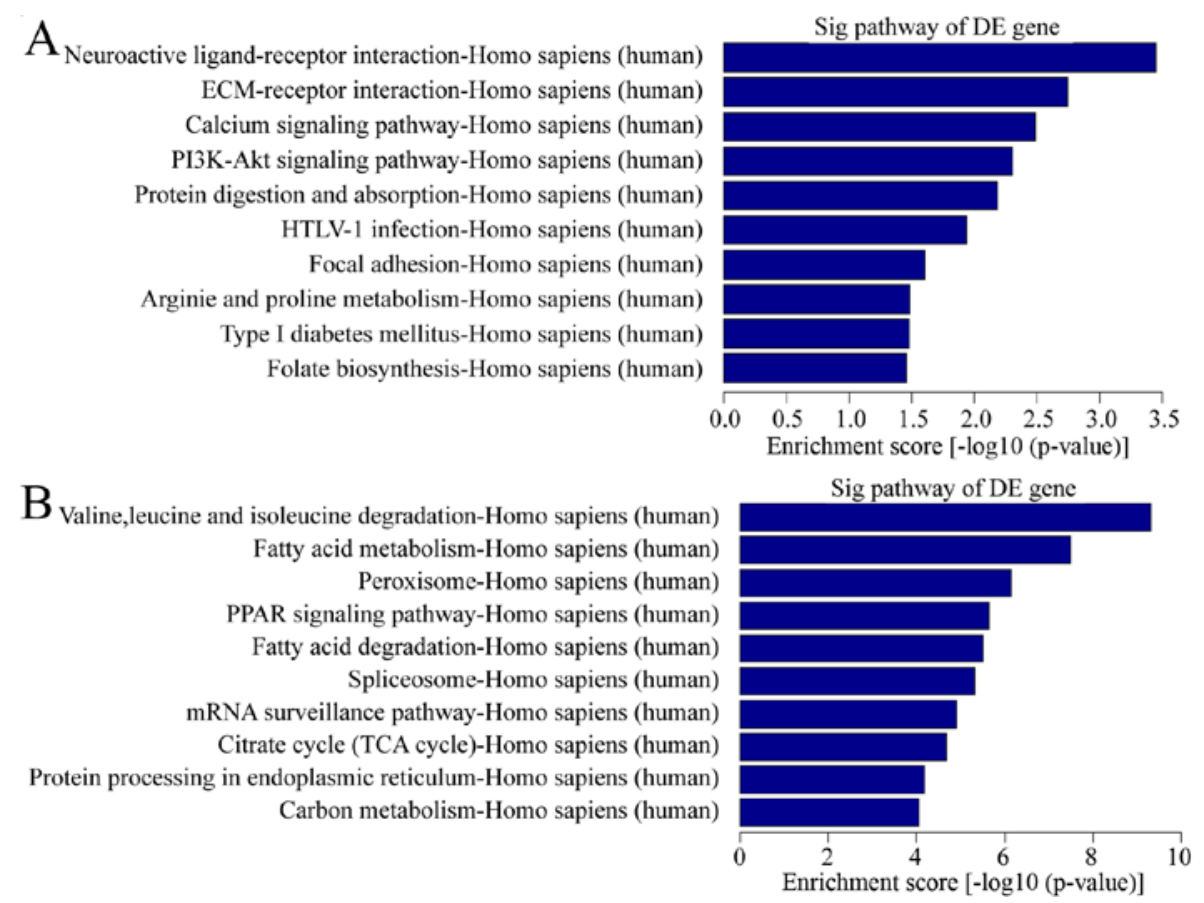

Figure 4. Pathway analysis. Pathway analysis is a functional analysis which maps genes to KEGG pathways. (A) Pathways related with upregulated transcripts in keloid. (B) Pathways related with downregulated transcripts in keloid. The bar plot shows the top ten enrichment score [-log10 (P-value)] value of the significant enrichment pathway. 


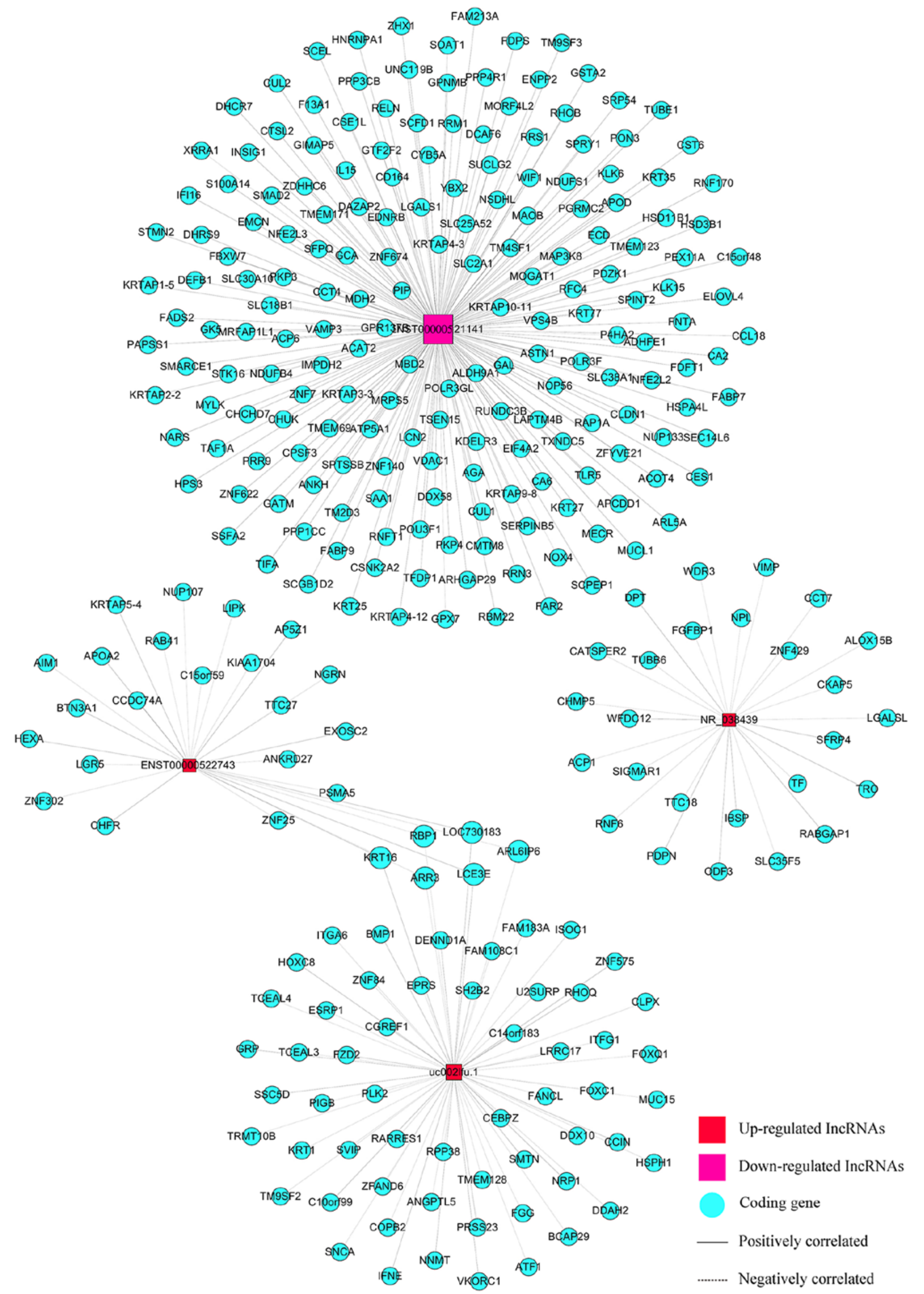

Figure 5. The co-expression network. Four selected lncRNAs were co-expression with 298 coding gene transcripts. NR_038439 expression correlates with 26 coding gene transcripts $(\mathrm{PCC}>0.999)$. 

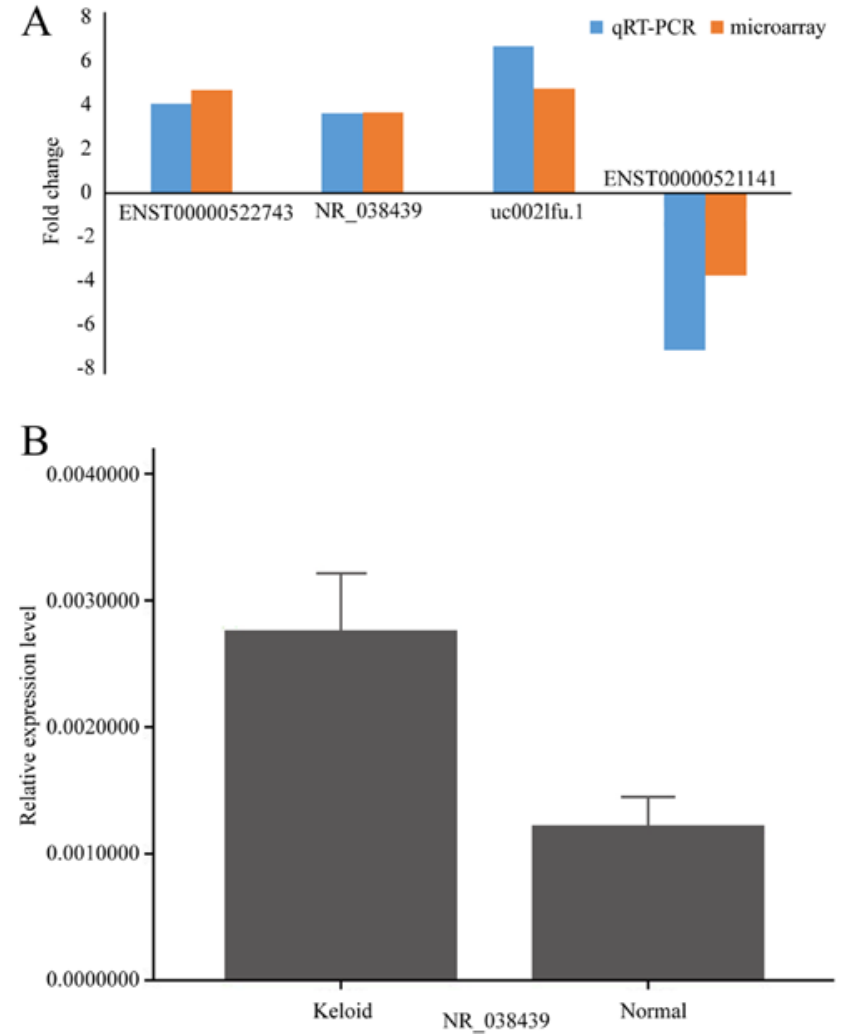

Figure 6. Relative expression of candidate lncRNAs in keloid and normal skin tissue validated by qRT-PCR. LncRNAs in keloid and normal skin tissue validated by qRT-PCR. (A) Comparison between microarray and qRT-PCR results. The columns in the chart represent the log-transformed median fold changes (keloid/normal skin) in expression among 3 pairs of samples $(\mathrm{P}<0.05)$. The qRT-PCR results and microarray data are consistent. (B) Validation of NR_038439 in 13 other paired tissues. The result was consistent.

research on IncRNA characterization will help us understand the mechanism of keloid formation, and enable development of personalized therapeutic strategies.

By performing microarray profiling, we found 16,710 IncRNAs and 18,788 mRNAs in keloid. Among them, 1,731 IncRNAs were constantly upregulated and 782 were downregulated, whereas, 1,079 mRNAs were consistently upregulated and 3,282 were downregulated. GO analysis showed that upregulated lncRNAs were enriched in biological regulation, while downregulated ones were enriched in cellular process and metabolic process. These also had a tight connection with keloid formation. Using KEGG pathway analysis, we found an obvious change in ECM-receptor interaction, calcium signaling pathway, focal adhesion and mRNA surveillance pathway.

After validation by qRT-PCR, IncRNA CACNA1G-AS1 was proven to have high expression in a total of 16 keloid tissues. CACNA1G-AS1 is an antisense RNA to CACNA1G. CACNA1G encodes Cav3.1, which is a subtype of T-type $\mathrm{Ca}^{2+}$ channels (28). Cav3.1 was found to be a tumor suppressor $(29,30)$. As far as we know, there are no reports on the function of CACNA1G-AS1. On the one hand, we have started to build models to explore its potential function. On the other hand, we are trying to find out how other antisense lncRNAs interact with their target genes, which may give us an inspiration on the current dilemma.
Antisense RNAs are defined as RNAs transcribed either from opposite strand of a protein coding gene or a sense strand-derived RNA (31). They can either positively or negatively modulate protein-coding genes (32). Xue et al (33) found that in bladder cancer cells, lncRNA MDC1-AS can inhibit malignant cell performance by upregulating the expression of $M D C 1$, a cancer suppressing gene, in both mRNA and protein levels. Our mRNA microarray result showed that CACNA1G also exhibited higher expression level in keloid. Thus, we presumed that CACNA1G-AS1 might regulate the pathological process in keloid through interacting with CACNA1G. More experiments are needed to explore the hypothesis. The L-type calcium channel blocker verapamil, has already been used in clinical treatment for keloid and proven effective (34). An 18-month follow-up of clinical study showed that in a group of patients who received intralesional verapamil hydrochloride injections after surgery, keloid was cured in 54\% of them, compared to $18 \%$ in the control group who received the same therapy except verapamil (35). Some researchers believe that hypertension is one of the reasons that may cause keloid, thus anti-hypertension pharmaceuticals such as verapamil is useful (36). Verapamil can also decrease the production of cytokines such as IL-6 and VEGF in central keloid fibroblasts, which are responsible for irregular proliferation of keloid fibroblasts (37). Based on these theories, we propose that Cav3.1 blocker may also be valid in treating keloid in a similar way.

Besides the CACNA1G, we found 26 other genes related to CACNA1G-AS1 in the co-expression network. They were RABGAP1, TTC18,PDPN, TUBB6,WDR3,ACP1, WFDC12, DPT, SIGMAR1, LGALSL, IBSP, ODF3, ZNF429, CHMP5, RNF6, ALOX15B, TF, SLC35F5, FGFBP1, NPL, VIMP, TRC, CCT7, SFRP4, CATSPER2 and CKAP5. Many of them are involved in cell proliferation and preventing cell apoptosis, which are commonly seen in keloid. Dermatopontin (DPT) participates in wound healing by activating fibronectin and improving cell adhesion (38). CHMP5, a multivesicular body, can inhibit cell apoptosis. When expression of CHMP5 is inhibited in leukemic cells, caspase-3 is activated, and apoptosis is stimulated (39). FGFBP1, known as FGFBP, is upregulated in several types of tumors and critical for tumor angiogenesis (40). More research should be done to establish related regulation mechanisms between these coding and noncoding genes.

In conclusion, we profiled differentially expressed lncRNAs and mRNAs in keloid and normal skin tissue. Further information on the candidate lncRNAs and their associated protein-coding genes were also studied. CACNA1G-AS1 was upregulated in keloid, and it may be important in keloid formation. However, further studies of the possible mechanism are required. Our findings indicate that IncRNAs are involved in the pathological process of keloid, which may help to explain the mechanism of keloid formation.

\section{Acknowledgements}

The present study was supported by a grant from the National Natural Science Foundation of China (NSFC) (no. 81071571). 


\section{References}

1. Halim AS, Emami A, Salahshourifar I and Kannan TP: Keloid scarring: Understanding the genetic basis, advances, and prospects. Arch Plast Surg 39: 184-189, 2012

2. van Leeuwen MC, van der Wal MB, Bulstra AE, GalindoGarre F, Molier J, van Zuijlen PP, van Leeuwen PA and Niessen FB: Intralesional cryotherapy for treatment of keloid scars: A prospective study. Plast Reconstr Surg 135: 580-589, 2015.

3. Erol OO, Gurlek A, Agaoglu G, Topcuoglu E and Oz H: Treatment of hypertrophic scars and keloids using intense pulsed light (IPL). Aesthetic Plast Surg 32: 902-909, 2008.

4. Juckett $G$ and Hartman-Adams H: Management of keloids and hypertrophic scars. Am Fam Physician 80: 253-260, 2009.

5. Mseddi M, Mesrati H, Ktaari S, Amouri M, Chaaben H, Boudaya S and Turki H: Treatment of keloid with phenol: A new therapy. Ann Dermatol Venereol 141: 493-499, 2014 (In French).

6. van Leeuwen MC, Bulstra AE, van Leeuwen PA and Niessen FB: A new argon gas-based device for the treatment of keloid scars with the use of intralesional cryotherapy. J Plast Reconstr Aesthet Surg 67: 1703-1710, 2014

7. Nakashima M, Chung S, Takahashi A, Kamatani N, Kawaguchi T, Tsunoda T, Hosono N, Kubo M, Nakamura Y and Zembutsu H: A genome-wide association study identifies four susceptibility loci for keloid in the Japanese population. Nat Genet 42: 768-771, 2010.

8. Chen W, Fu X, Sun X, Sun T, Zhao Z and Sheng Z: Analysis of differentially expressed genes in keloids and normal skin with cDNA microarray. J Surg Res 113: 208-216, 2003

9. Unahabhokha T, Sucontphunt A, Nimmannit U, Chanvorachote P, Yongsanguanchai N and Pongrakhananon V: Molecular signalings in keloid disease and current therapeutic approaches from natural based compounds. Pharm Biol 53: 457-463, 2015.

10. Murao N, Seino K, Hayashi T, Ikeda M, Funayama E, Furukawa H, Yamamoto Y and Oyama A: Treg-enriched CD4 $\mathrm{T}$ cells attenuate collagen synthesis in keloid fibroblasts. Exp Dermatol 23: 266-271, 2014

11. Kung JT, Colognori D and Lee JT: Long noncoding RNAs: Past, present, and future. Genetics 193: 651-669, 2013.

12. Bonasio R and Shiekhattar R: Regulation of transcription by long noncoding RNAs. Annu Rev Genet 48: 433-455, 2014.

13. He Y, Meng XM, Huang C, Wu BM, Zhang L, Lv XW and Li J: Long noncoding RNAs: Novel insights into hepatocelluar carcinoma. Cancer Lett 344: 20-27, 2014.

14. Martens-Uzunova ES, Böttcher R, Croce CM, Jenster G, Visakorpi T and Calin GA: Long noncoding RNA in prostate, bladder, and kidney cancer. Eur Urol 65: 1140-1151, 2014.

15. Hombach $\mathrm{S}$ and Kretz M: The non-coding skin: Exploring the roles of long non-coding RNAs in epidermal homeostasis and disease. BioEssays 35: 1093-1100, 2013.

16. Kretz M, Webster DE, Flockhart RJ, Lee CS, Zehnder A, LopezPajares V, Qu K, Zheng GX, Chow J, Kim GE, et al: Suppression of progenitor differentiation requires the long noncoding RNA ANCR. Genes Dev 26: 338-343, 2012.

17. Boggio RF, Freitas VM, Cassiola FM, Urabayashi M and Machado-Santelli GM: Effect of a calcium-channel blocker (verapamil) on the morphology, cytoskeleton and collagenase activity of human skin fibroblasts. Burns 37: 616-625, 2011.

18. Kashiyama K, Mitsutake N, Matsuse M, Ogi T, Saenko VA, Ujifuku K, Utani A, Hirano A and Yamashita S: miR-196a downregulation increases the expression of type I and III collagens in keloid fibroblasts. J Invest Dermatol 132: 1597-1604, 2012.

19. Liu Y, Yang D, Xiao Z and Zhang M: miRNA expression profiles in keloid tissue and corresponding normal skin tissue. Aesthetic Plast Surg 36: 193-201, 2012.

20. Liu Y, Wang X, Yang D, Xiao Z and Chen X: MicroRNA-21 affects proliferation and apoptosis by regulating expression of PTEN in human keloid fibroblasts. Plast Reconstr Surg 134: 561e-573e, 2014
21. Di Gesualdo F, Capaccioli S and Lulli M: A pathophysiological view of the long non-coding RNA world. Oncotarget 5: 10976-10996, 2014.

22. Kretz M: TINCR, staufen 1 , and cellular differentiation. RNA Biol 10: 1597-1601, 2013.

23. Bikle DD: The vitamin D receptor: A tumor suppressor in skin. Discov Med 11: 7-17, 2011.

24. Jiang YJ and Bikle DD: LncRNA profiling reveals new mechanism for VDR protection against skin cancer formation. J Steroid Biochem Mol Biol 144pa: 87-90, 2014.

25. Wan DC and Wang KC: Long noncoding RNA: Significance and potential in skin biology. Cold Spring Harb Perspect Med 4: 1-10, 2014.

26. Mazar J, Zhao W, Khalil AM, Lee B, Shelley J, Govindarajan SS, Yamamoto F, Ratnam M, Aftab MN, Collins S, et al: The functional characterization of long noncoding RNA SPRY4-IT1 in human melanoma cells. Oncotarget 5: 8959-8969, 2014.

27. Wang KC and Chang HY: Molecular mechanisms of long noncoding RNAs. Mol Cell 43: 904-914, 2011.

28. Fukunaga K: Cognitive Function and Calcium. Cognitive improvement through $\mathrm{T}$ type calcium channel stimulation. Clin Calcium 25: 247-254, 2015 (In Japanese).

29. Toyota M, Ho C, Ohe-Toyota M, Baylin SB and Issa JP: Inactivation of CACNA1G, a T-type calcium channel gene, by aberrant methylation of its $5^{\prime} \mathrm{CpG}$ island in human tumors. Cancer Res 59: 4535-4541, 1999.

30. Ohkubo T and Yamazaki J: T-type voltage-activated calcium channel Cav3.1, but not Cav3.2, is involved in the inhibition of proliferation and apoptosis in MCF-7 human breast cancer cells. Int J Oncol 41: 267-275, 2012.

31. Villegas VE and Zaphiropoulos PG: Neighboring gene regulation by antisense long non-coding RNAs. Int J Mol Sci 16: 3251-3266, 2015.

32. Zhang ZZ, Shen ZY, Shen YY, Zhao EH, Wang M, Wang CJ, $\mathrm{Cao} \mathrm{H}$ and $\mathrm{Xu}$ J: HOTAIR long noncoding RNA promotes gastric cancer metastasis through suppression of Poly $\mathrm{r}(\mathrm{C})$ Binding Protein (PCBP) 1. Mol Cancer Ther 14: 1162-1170, 2015.

33. Xue Y, Ma G, Zhang Z, Hua Q, Chu H, Tong N, Yuan L, Qin C, Yin $\mathrm{C}$, Zhang $\mathrm{Z}$, et al: A novel antisense long noncoding RNA regulates the expression of MDC1 in bladder cancer. Oncotarget 6: 484-493, 2015

34. Ledon JA, Savas J, Franca K, Chacon A and Nouri K: Intralesional treatment for keloids and hypertrophic scars: A review. Dermatol Surg 39: 1745-1757, 2013.

35. D'Andrea F, Brongo S, Ferraro G and Baroni A: Prevention and treatment of keloids with intralesional verapamil. Dermatology 204: 60-62, 2002.

36. Huang $\mathrm{C}$ and Ogawa R: Pharmacological treatment for keloids. Expert Opin Pharmacother 14: 2087-2100, 2013.

37. Giugliano G, Pasquali D, Notaro A, Brongo S, Nicoletti G, D'Andrea F, Bellastella A and Sinisi AA: Verapamil inhibits interleukin- 6 and vascular endothelial growth factor production in primary cultures of keloid fibroblasts. Br J Plast Surg 56: 804-809, 2003.

38. Wu W, Okamoto O, Kato A, Matsuo N, Nomizu M, Yoshioka H and Fujiwara S: Dermatopontin regulates fibrin formation and its biological activity. J Invest Dermatol 134: 256-263, 2014.

39. Wang H, Liu J, Wang F, Chen M, Xiao Z, Ouyang R, Fei A, Shen Y and Pan S: The role of charged multivesicular body protein 5 in programmed cell death in leukemic cells. Acta Biochim Biophys Sin (Shanghai) 45: 383-390, 2013.

40. Abuharbeid S, Czubayko F and Aigner A: The fibroblast growth factor-binding protein FGF-BP. Int $\mathrm{J}$ Biochem Cell Biol 38: 1463-1468, 2006. 